\title{
Utilização da tração halo-craniana pré-operatória no tratamento de deformidades vertebrais de alto valor angular
}

Halo gravity preoperative traction in severe spine deformities treatment

\author{
Uso de tracción halo gravitacional en el tratamiento quirúrgico de \\ las deformidades graves de la columna vertebral
}

\author{
Maximiliano Aguiar Porto' \\ Carlos Fernando Pereira da Silva Herrero² \\ Marcello Henrique Nogueira-Barbosa ${ }^{3}$ \\ Helton LA Defino ${ }^{4}$
}

\section{RESUMO}

Objetivos: avaliar o resultado do tratamento cirúrgico das deformidades acentuadas da coluna vertebral por meio da utilização da tração halogravitacional. Métodos: foram estudados 17 pacientes portadores de diferentes patologias ( 6 congênitas, 2 pós-neoplasia, 2 neurofibromatoses, 1 idiopática, 1 raquitismo e 5 neuromusculares) submetidos a cirurgia para correção de deformidade de alto valor angular na coluna vertebral. A idade no momento da cirurgia variou de 6 a 26 anos (média de 13,75 anos $\pm 4,5)$. Os parâmetros clínicos avaliados foram o sexo, a idade, o tempo e o peso da tração. Os parâmetros radiográficos estudados foram: a medida da escoliose, da cifose e a altura da coluna vertebral. Os parâmetros radiográficos foram avaliados no período pré-operatório, durante a tração, e no pós-operatório. Resultados: o tempo de tração variou de 14

\section{ABSTRACT}

Objective: evaluate the surgical treatment results of severe spine deformities using the preoperative halo gravity traction. Methods: seventeen scoliosis patients with different pathologies were evaluated (6 congenital, 2 tumor, 2 neurofibromatosis, 1 idiopathic, 1 rickets, and 5 neuromuscular). Those patients underwent a deformity correction surgery in the spine. The variety of age was 6 to 26 years-old (average of 13.75 \pm 4.5 ). The clinical features evaluated were age, gender, time of traction and weight used on traction. The radiographic data analyzed follows: scoliosis curve kyphosis, and spine height. Those features were evaluated in the preoperative, during traction and postoperative period. Results: the time of traction range was of 14 to 106 days (34.5 \pm 20.83$)$ and the weight of traction percentage to the

\section{RESUMEN}

Objetivos: evaluar el resultado del tratamiento quirúrgico en deformidades graves de la columna vertebral con el uso de tracción halo gravitacional. Métodos: fueron estudiados 17 pacientes portadores de diferentes patologías (seis congénitas, dos post neoplasia, dos neurofibromatosis, un idiopático, un raquitismo, cinco neuromusculares) sometidos a cirugía para corrección de deformidades graves de la columna vertebral. La edad en el momento de la cirugía variaba de 6 a 26 años (media de 13,75 años $\pm 4,5)$. Los parámetros clínicos analizados fueron: sexo, edad, tiempo y peso en la tracción y complicaciones clínicas. Los parámetros radiográficos estudiados fueron: medida de la escoliosis, cifosis, altura de la columna vertebral. Los parámetros radiográficos fueron evaluados en el período preoperatorio y post-ope-

\footnotetext{
*Trabalho realizado no Departamento de Biomecânica, Medicina e Reabilitação do Aparelho Locomotor - Hospital das Clínicas da Faculdade de Medicina de Ribeirão Preto da Universidade de São Paulo - USP - Ribeirão Preto (SP), Brasil.

1 Pós-graduando (Doutorado) do Departamento de Biomecânica, Medicina e Reabilitação do Aparelho Locomotor do Hospital das Clínicas da Faculdade de Medicina de Ribeirão Preto da Universidade de São Paulo - USP - Ribeirão Preto (SP), Brasil.

${ }^{2}$ Médico assistente do Departamento de Biomecânica, Medicina e Reabilitação do Aparelho Locomotor do Hospital das Clínicas da Faculdade de Medicina de Ribeirão Preto da Universidade de São Paulo - USP - Ribeirão Preto (SP), Brasil.

${ }^{3}$ Doutor; Professor da Divisão de Radiologia do Departamento de Clínica Médica da Faculdade de Medicina de Ribeirão Preto da Universidade de São Paulo USP - Ribeirão Preto (SP), Brasil.

${ }^{4}$ Doutor; Professor Titular do Departamento de Biomecânica, Medicina e Reabilitação do Aparelho Locomotor do Hospital das Clínicas da Faculdade de Medicina de Ribeirão Preto da Universidade de São Paulo - USP - Ribeirão Preto (SP), Brasil.
} 
a $106 \operatorname{dias}(34,5 \pm 20,83)$ e a porcentagem de peso da tração em relação ao peso corporal variou de 12,5 a $50 \%$ $(30 \% \pm 9,36)$. A média inicial das escolioses foi de $98,6 \pm 25,7^{\circ}$ (medida Cobb), reduzido para $83,5^{\circ} \pm 28,5^{\circ} \mathrm{du}-$ rante a tração e para $77^{\circ} \pm 29,4^{\circ}$ no pósoperatório, com a média de correção de $27^{\circ}$, sendo $63 \%$ destes ocorridos no período do uso do halo. A média inicial da cifose foi de $86,3 \pm 32,08^{\circ}$ (Cobb), reduzido para $62,2^{\circ} \pm 19,9^{\circ}$ durante a tração e para $60^{\circ} \pm 20,9^{\circ}$ no pós-operatório, com a média de correção de $26^{\circ}$, sendo $92 \%$ durante a tração. Conclusão: o tratamento cirúrgico das deformidades acentuadas da coluna vertebral, por meio da tração halo-gravitacional pré-operatória, é um método que melhora o ângulo de Cobb reduzindo a correção aguda intraoperatória assim como seus riscos de lesão neurológica. patient's body weight varied of 12.5 to $50 \%(30 \% \pm 9.36)$. The average value for scoliosis was 98.6 $25.7^{\circ}$ (Cobb) before the surgery and $77^{\circ} \pm 29.4^{\circ}$ postoperative, showing the correction average of $27^{\circ}$ with $63 \%$ of the correction during the traction time. The kyphosis average was $86.3 \pm 32.08^{\circ}$ (Cobb) before and $60^{\circ} \pm 20.9^{\circ}$ after surgery, representing the correction of $26^{\circ}$, with $92 \%$ of the correction in the traction time. Conclusion: the surgical treatment of severe spine deformities through the preoperative halo gravity traction brings good correction for the curves, thus reducing the amount of intraoperative correction. ratorio. Resultados: el tiempo de tracción varió de 14 hasta 106 días (34.5 20.83$)$ y el porcentaje de peso de la tracción, en relación al peso corporal, varió del 12,5 hasta el $50 \%$ (30\% 99.36). El valor medio de la escoliosis era de $98.6 \pm 25.7^{\circ}$ (Cobb) antes de la cirugía y de $77 \pm 29.4^{\circ}$ en el post-operatorio, con una media de corrección de $25^{\circ}$. El valor medio de la cifosis era de $86.3 \pm 32.08^{\circ}$ (Cobb) antes de la cirugía y de 60 ${ }^{\circ} \pm 20.9^{\circ}$ en el post-operatorio, con una media de corrección de $26^{\circ}$. Conclusión: el tratamiento quirúrgico de las deformidades graves de la columna vertebral, por medio de la tracción halo gravitacional, mostró buena corrección de la deformidad, reduciendo la corrección aguda intraoperatoria.
DESCRITORES: Escoliose; Cifose; Tração
KEYWORDS: Scoliosis;

Kyphosis; Traction
DESCRIPTORES: Escoliosis; Cifosis; Tracción

\section{INTRODUÇÃO}

$\mathrm{O}$ tratamento cirúrgico das deformidades graves e com alto valor angular da coluna vertebral representa um grande desafio devido às dificuldades intrínsecas para a sua correção ao risco de lesão neurológica associado. Além disso, em nosso meio, faltam recursos à monitoração intraoperatória dos pacientes, o que limita a utilização de osteotomias ou ressecções vertebrais na correção das deformidades. $\mathrm{O}$ risco de lesão neurológica pode aumentar na presença de história pregressa de alteração intraespinhal ou cirurgia prévia $^{1,2}$.

Métodos de tração pré-operatória foram descritos para permitir correção gradual das deformidades, reduzindo as complicações de um procedimento corretivo agudo. As vantagens da tração são: menor solicitação mecânica submetida à interface osso-implante no momento da cirurgia definitiva por ser realizada em uma curva de menor valor angular e, uma vez que o paciente se encontra alerta durante o período de tração, possibilidade de avaliação contínua dos sintomas neurológicos, permitindo intervenção imediata, na eventualidade de sua ocorrência² ${ }^{2}$.

Dentre os métodos de tração descritos destacam-se a halo pélvica, a halo femoral e a halo gravitacional. Em contraste à tração halo-pélvica e halo-femoral, a tração halo-gravitacional, popularizada por Stagnara, utiliza o peso corporal do paciente como uma contra força e não requer restrição prolongada no leito, permitindo a ele locomover-se por meio de cadeira de rodas ou andar com apoio especial ${ }^{1-3}$.

O objetivo deste estudo foi avaliar o uso da tração halocraniana pré-operatória em pacientes com deformidades acentuadas da coluna vertebral e submetidos ao tratamento cirúrgico sem a monitoração intraoperatória das estruturas nervosas e realização de osteotomias ou ressecções vertebrais.

\section{MÉTODOS}

Foram avaliados 17 pacientes com deformidades de alto valor angular da coluna vertebral (10 do sexo feminino e 7 do sexo masculino) com idade variando 6 e 26 anos (média de 13,75 4 ,52 anos). Estes foram submetidos ao tratamento cirúrgico após período de tração. A etiologia das deformidades era neuromuscular em cinco pacientes, congênita em seis pacientes, neurofibromatose em dois pacientes, sequela de neoplasia intraraquídea em dois pacientes, idiopática em um paciente e raquitismo em um paciente. As características gerais dos pacientes estão ilustradas na tabela 1.

Todos os pacientes foram submetidos à instalação do halo craniano de acordo com a técnica descrita por Mubarak et $a l^{4}$. A tração foi iniciada 24 horas após a inserção dos pinos com $1 \mathrm{~kg}$ e aumentada com $1 \mathrm{~kg}$ por dia, 
TABELA 1 - Dados clínicos e cirúrgicos

\begin{tabular}{|c|c|c|c|c|c|c|}
\hline Paciente & Sexo & Idade & Etiologia & $\begin{array}{c}\text { Tempo de } \\
\text { tração (dias) }\end{array}$ & Cirurgia & $\begin{array}{c}\text { Peso tração } \\
(\mathrm{kg})\end{array}$ \\
\hline 1 & $\mathrm{~F}$ & 8 & $\mathrm{NF}+$ Escoliose & 20 & AVP & 26 \\
\hline 2 & $\mathrm{~F}$ & 21 & Cifoescoliose + Miastenia & 30 & AVP & 38.5 \\
\hline 3 & $\mathrm{~F}$ & 17 & Escoliose Neuromuscular & 36 & AVP & 36.2 \\
\hline 4 & M & 13 & Cifoescoliose pós ressecção de astrocitoma & 106 & $\mathrm{LP}+\mathrm{AVP}+\mathrm{AVA}$ & 34 \\
\hline 5 & $\mathrm{~F}$ & 11 & Escoliose Congênita & 27 & AVP & 24 \\
\hline 6 & M & 26 & Cifoescoliose + Astrocitoma & 38 & AVP & 70 \\
\hline 7 & M & 17 & Cifoescoliose congênita + Sd Alcoólica Fetal & 40 & AVP & 49 \\
\hline 8 & M & 11 & Escoliose Congênita & 55 & AVP & 16.7 \\
\hline 9 & M & 14 & Cifoescoliose Congênita & 27 & $\mathrm{AVP}+\mathrm{AVA}$ & 41 \\
\hline 10 & $\mathrm{~F}$ & 6 & Cifoescoliose + Larsen & 27 & $\mathrm{AVA}+\mathrm{AVP}$ & 15 \\
\hline 11 & M & 15 & Cifoescoliose $+M M C$ & 21 & $\mathrm{AVA}+\mathrm{AVP}$ & 40 \\
\hline 12 & $\mathrm{~F}$ & 11 & Escoliose idiopática infantil & 20 & $\mathrm{LA}+\mathrm{LP}+\mathrm{AVP}+\mathrm{AVA}$ & 21 \\
\hline 13 & $\mathrm{~F}$ & 22 & Cifoescoliose + Raquitismo & 14 & LP+AVA c/fíbula & 38 \\
\hline 14 & $\mathrm{~F}$ & 16 & Cifoescoliose congênita + Siringomielia & 22 & AVP & 48 \\
\hline 15 & $\mathrm{~F}$ & 13 & Cifoescoliose congênita & 35 & AVA c/fíbula+AVP & 22 \\
\hline 16 & $\mathrm{~F}$ & 13 & Escolise +NPSMP & 36 & $\mathrm{AVP}+\mathrm{AVA}$ & 21 \\
\hline 17 & M & 14 & Cifoescoliose+NF & 34 & AVP & 42 \\
\hline
\end{tabular}

NPSMP: Neuropatia sensitivo-motora periférica; MMC: mielomeningocele; NF: neurofibromatose; AVA: artrodese via anterior; AVP: artrodese via posterior; LP: liberação posterior; LA: liberação anterior.

de acordo com a tolerância de cada paciente e a presença de sintomas neurológicos que eram avaliados diariamente. Os pinos foram checados de $48 \mathrm{em} 48$ horas e, quando necessário, reapertados. $\mathrm{O}$ paciente permanecia em tração contínua em decúbito dorsal ou na posição sentada em cadeira de Stagnara (Figura1).
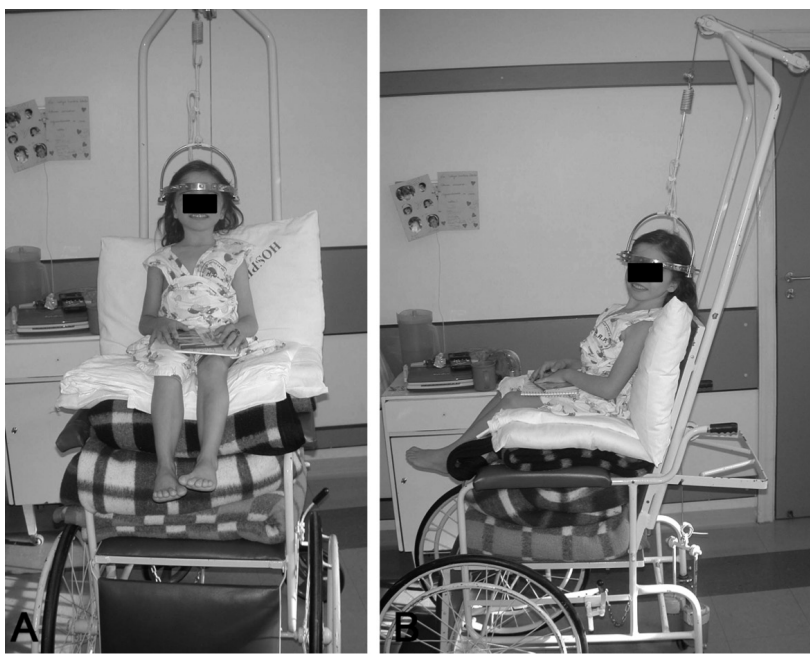

Figura 1

Fotografia ilustrando paciente com halo-craniano na cadeira especial.

O tratamento cirúrgico foi realizado por meio da artrodese vertebral posterior isolada em nove pacientes e por meio da abordagem combinada (anterior e posterior) em oito pacientes. A abordagem anterior foi utilizada no intuito de aumentarmos a correção das deformidades por meio da liberação de partes moles e realizar a artrodese anterior nos casos com maior rigidez.

Os pacientes foram avaliados por meio de parâmetros clínicos: idade, sexo, patologia, tempo de tração e peso de tração; e radiológicos: medida da escoliose, cifose e lordose pelo método de Cobb. Os parâmetros foram avaliados no período pré-operatório, durante a tração e pós-operatório.

\section{RESULTADOS}

O tempo de permanência na tração halo-craniana variou de 14 a 106 dias (média de 34,5 20,83 ) e o peso máximo utilizado na tração variou de 5 a $14,5 \mathrm{~kg}(9,33 \pm 3,61)$. O peso utilizado representou, em média, $30 \%$ do peso corporal dos pacientes medido no início da tração (Tabela 1).

A medida da curva principal da escoliose no período pré-operatório variou de $45^{\circ}$ a $163^{\circ}\left(98,6 \pm 35,7^{\circ}\right)$, de $27^{\circ}$ a $144^{\circ}\left(83,5 \pm 28,5^{\circ}\right)$ durante a tração e de $19^{\circ}$ a $135^{\circ}$ $\left(77 \pm 29,4^{\circ}\right)$ no período pós-operatório (Figuras 2 e 3 ). A correção da curva principal da escoliose variou de $0^{\circ}$ a $34^{\circ}$ $\left(17,58 \pm 12,8^{\circ}\right) \mathrm{com}$ a tração halo-gravitacional e de $8^{\circ}$ a $70^{\circ}$ $\left(31,1 \pm 16,5^{\circ}\right)$ no período pós-operatório. O percentual de

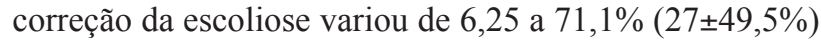
sendo $63 \%$ obtidos durante o período de tração (Tabelas 2 e 3 ).

Os valores da curva secundária da escoliose no período pré-operatório variaram de $25^{\circ}$ a $110^{\circ}$ $\left(59,1 \pm 21,44^{\circ}\right)$, de $8^{\circ}$ a $109^{\circ}\left(53,1 \pm 25,8^{\circ}\right)$ durante a tração e de $4^{\circ}$ a $108^{\circ}\left(49,6 \pm 28,3^{\circ}\right)$ no período pós-operatório (Figura 2). A correção da curva secundária da escoliose variou de $0^{\circ}$ a $23^{\circ}\left(10,4 \pm 8,8^{\circ}\right)$ com a tração halo-gravitacional e de $0^{\circ}$ a $44^{\circ}\left(18,35 \pm 12,6^{\circ}\right)$ no período pós-operatório (Tabelas 2 e 3 ). 

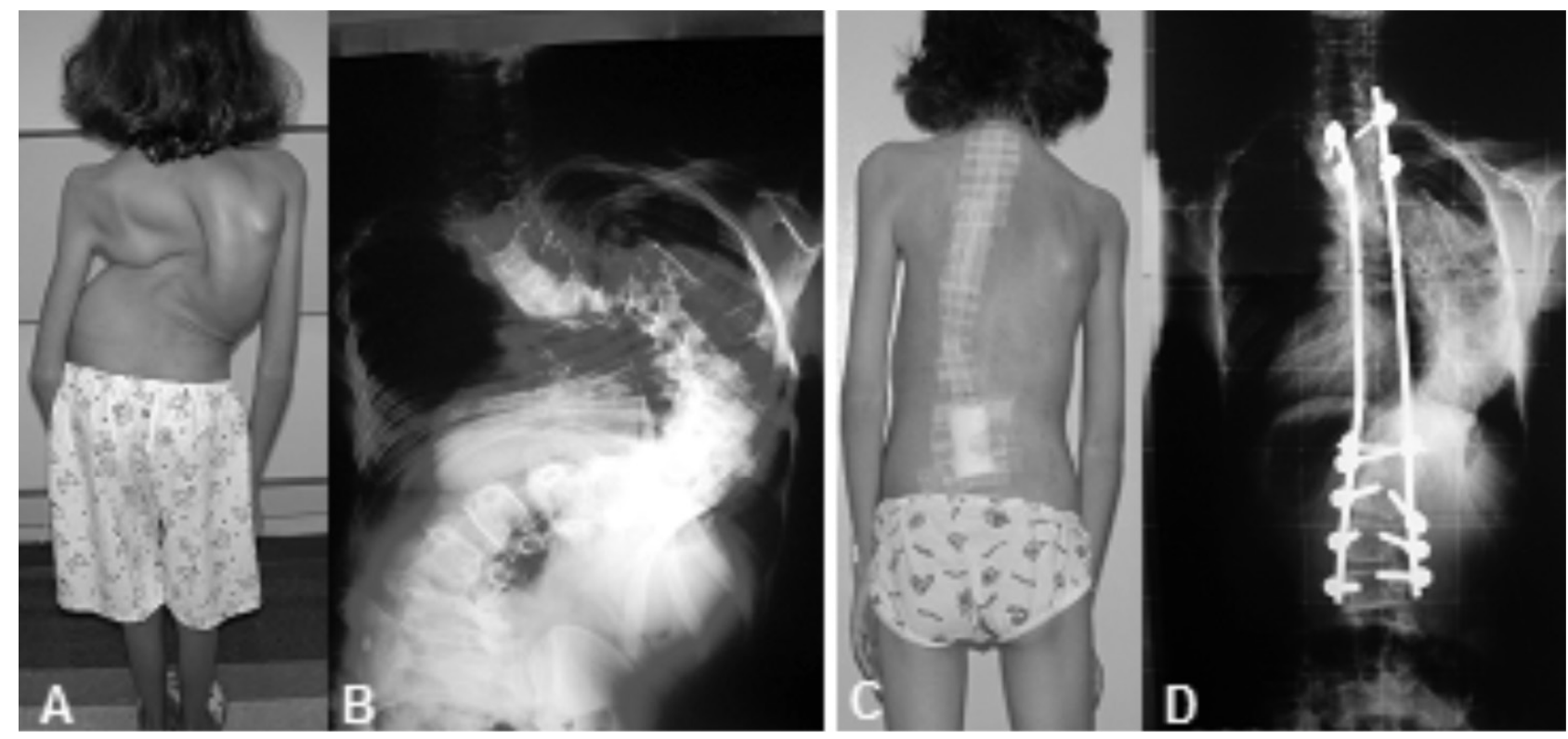

Figura 2

Fotografia pré ( $A$ e B) e pós-operatória ( $C$ e D) de paciente com escoliose congênita (Paciente $n^{\circ}$ 5).
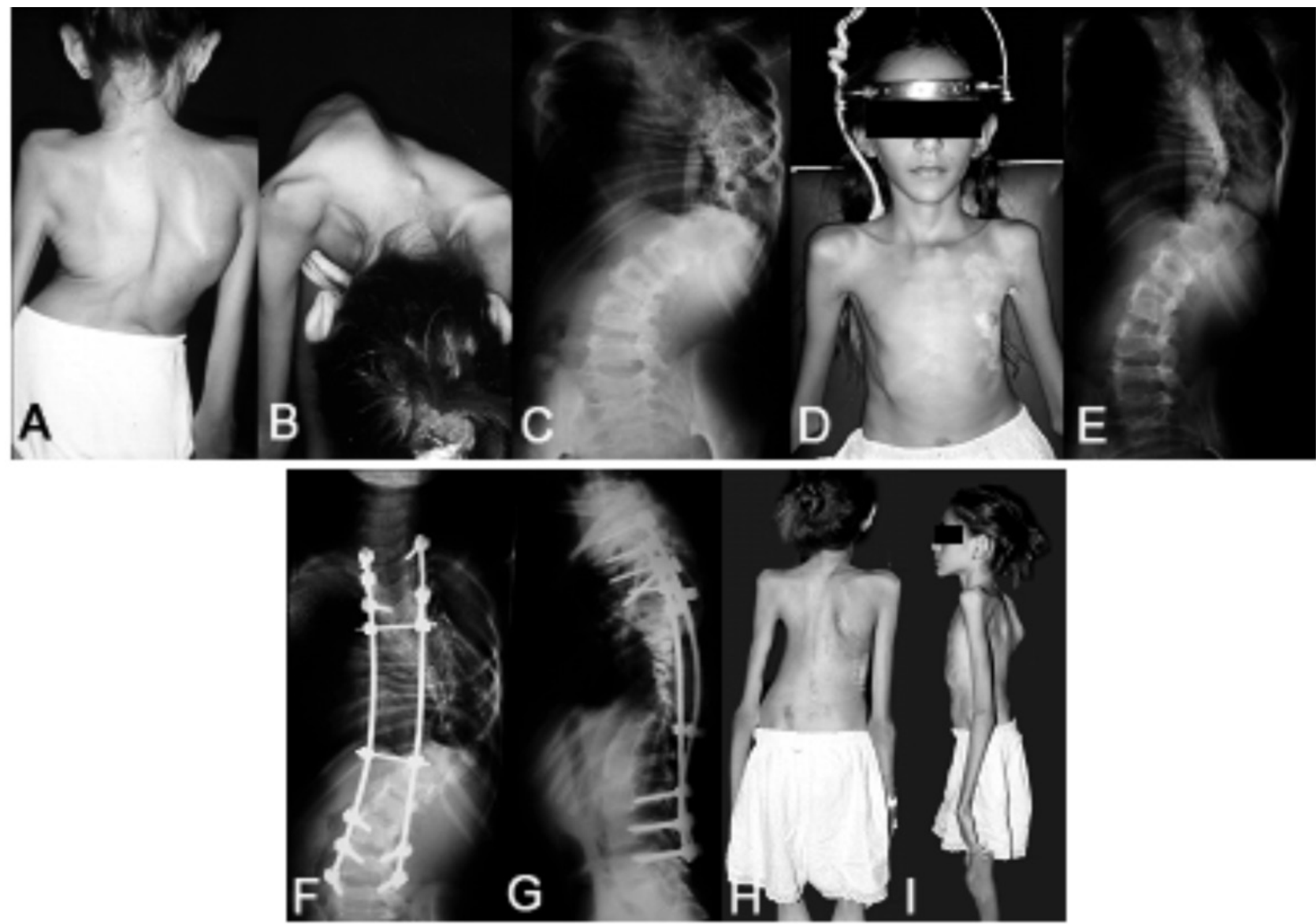

Figura 3

Fotografias pré-operatórias $(A, B$ e $C)$, durante tração $(D$ e $E)$ e pós-operatórias $(F, G, H, I)$ de paciente portadora de escoliose infantil (Paciente $\left.n^{\circ} 12\right)$. 
TABELA 2 - Valores e desvio-padrão nos diferentes quesitos e tempos

\begin{tabular}{lccc}
\hline & Curva principal & Cifose torácica & Altura da coluna \\
\hline Pré-operatório inicial & $98,6 \pm 35,7^{\circ}$ & $86,3 \pm 32^{\circ}$ & $30,6 \pm 6,86 \mathrm{~cm}$ \\
Tração pré-operatória & $83,5 \pm 28,5^{\circ *}$ & $62,2 \pm 19,9^{\circ * *}$ & $33,5 \pm 6,2 \mathrm{~cm} * *$ \\
Pós-operatório & $77 \pm 29,4^{\circ *}$ & $60,3 \pm 20,9^{\circ}$ & $36,3 \pm 6,5 \mathrm{~cm}$ \\
\hline
\end{tabular}

* $63 \%$ da correção; ${ }^{* *} 92 \%$ da correção; ${ }^{* *} 50,8 \%$ da correção. Tais valores significam a porcentagem de correção atribuída ao período de tração.

TABELA 3 - Descrição dos valores observados nos diferentes parâmetros radiográficos

\begin{tabular}{|c|c|c|c|c|c|c|c|c|}
\hline Paciente & $\begin{array}{l}\text { Curva } \\
\text { principal } \\
\text { inicial }\end{array}$ & $\begin{array}{l}\text { Curva } \\
\text { principal } \\
\text { final }\end{array}$ & $\begin{array}{c}\text { Curva } \\
\text { secundária } \\
\text { inicial }\end{array}$ & $\begin{array}{c}\text { Curva } \\
\text { secundária } \\
\text { final }\end{array}$ & $\begin{array}{l}\text { Cifose } \\
\text { torácica } \\
\text { inicial }\end{array}$ & $\begin{array}{l}\text { Cifose } \\
\text { torácica } \\
\text { final }\end{array}$ & $\begin{array}{c}\text { Altura C7- } \\
\text { S1 inicial } \\
(\mathrm{cm})\end{array}$ & $\begin{array}{c}\text { Altura } \\
\text { C7-S1 final } \\
(\mathrm{cm})\end{array}$ \\
\hline 1 & $85^{\circ}$ & $43^{\circ}$ & $56^{\circ}$ & $12^{\circ}$ & $22^{\circ}$ & $33^{\circ}$ & 33 & 39 \\
\hline 2 & $90^{\circ}$ & $52^{\circ}$ & $43^{\circ}$ & $21^{\circ}$ & $28^{\circ}$ & $6^{\circ}$ & 45 & 44 \\
\hline 3 & $45^{\circ}$ & $19^{\circ}$ & $25^{\circ}$ & $4^{\circ}$ & $8^{\circ}$ & $7^{\circ}$ & 46 & 46 \\
\hline 4 & $78^{\circ}$ & $22^{\circ}$ & $47^{\circ}$ & $17^{\circ}$ & $28^{\circ}$ & $17^{\circ}$ & 30 & 45 \\
\hline 5 & $163^{\circ}$ & $93^{\circ}$ & $61^{\circ}$ & $27^{\circ}$ & $77^{\circ}$ & $57^{\circ}$ & 28 & 39 \\
\hline 6 & $92^{\circ}$ & $65^{\circ}$ & $30^{\circ}$ & $6^{\circ}$ & $87^{\circ}$ & $48^{\circ}$ & 38 & 44 \\
\hline 7 & $114^{\circ}$ & $78^{\circ}$ & $110^{\circ}$ & $85^{\circ}$ & $63^{\circ}$ & $60^{\circ}$ & 32 & 39 \\
\hline 8 & $69^{\circ}$ & $34^{\circ}$ & $60^{\circ}$ & $34^{\circ}$ & $96^{\circ}$ & $59^{\circ}$ & 21 & 29 \\
\hline 9 & $110^{\circ}$ & $102^{\circ}$ & $72^{\circ}$ & $71^{\circ}$ & $80^{\circ}$ & $61^{\circ}$ & 27 & 37 \\
\hline 10 & $84^{\circ}$ & $60^{\circ}$ & $45^{\circ}$ & $30^{\circ}$ & $115^{\circ}$ & $94^{\circ}$ & 17 & 24 \\
\hline 11 & $144^{\circ}$ & $135^{\circ}$ & $103^{\circ}$ & $101^{\circ}$ & $46^{\circ}$ & $43^{\circ}$ & 33 & 33 \\
\hline 12 & $130^{\circ}$ & $106^{\circ}$ & $60^{\circ}$ & $44^{\circ}$ & $75^{\circ}$ & $78^{\circ}$ & 26 & 33 \\
\hline 13 & $85^{\circ}$ & $72^{\circ}$ & $63^{\circ}$ & $64^{\circ}$ & $100^{\circ}$ & $40^{\circ}$ & 35 & 38 \\
\hline 14 & $88^{\circ}$ & $44^{\circ}$ & $71^{\circ}$ & $55^{\circ}$ & $70^{\circ}$ & $40^{\circ}$ & 35 & 42 \\
\hline 15 & $66^{\circ}$ & $65^{\circ}$ & $44^{\circ}$ & $50^{\circ}$ & $130^{\circ}$ & $80^{\circ}$ & 27 & 35 \\
\hline 16 & $95^{\circ}$ & $57^{\circ}$ & $33^{\circ}$ & $14^{\circ}$ & $45^{\circ}$ & $50^{\circ}$ & 38 & 44 \\
\hline 17 & $97^{\circ}$ & $71^{\circ}$ & $54^{\circ}$ & $32^{\circ}$ & $110^{\circ}$ & $58^{\circ}$ & 34 & 42 \\
\hline
\end{tabular}

A medida da cifose no período pré-operatório variou de $8^{\circ}$ a $115^{\circ}\left(86,3 \pm 32^{\circ}\right)$, de $35^{\circ}$ a $85^{\circ}\left(62,2 \pm 19,9^{\circ}\right)$ durante a tração e de $7^{\circ}$ a $94^{\circ}\left(60,3 \pm 20,9^{\circ}\right)$ no período pós-operatório (Figura 4). A correção da cifose variou de $0^{\circ}$ a $51^{\circ}$ $\left(23,5 \pm 15,2^{\circ}\right)$ com a tração halo-gravitacional e de $0^{\circ}$ a $60^{\circ}$ $\left(21,5 \pm 20^{\circ}\right)$ no período pós-operatório. O percentual de correção da cifose variou de 0 a 47,2\% (28,2 $22,9 \%)$, com $92 \%$ da correção ocorrendo no período da tração (Tabela 2 e Figura 5).

A medida de altura da coluna vertebral variou de 17 a $46 \mathrm{~cm}(30,6 \pm 6,86)$ no período pré-operatório, de 22 a 42 $\mathrm{cm}(33,5 \pm 6,2)$ durante o período de tração, e de 24 a $46 \mathrm{~cm}$ $(36,3 \pm 6,5)$ no período pós-operatório. $\mathrm{O}$ aumento da altura da coluna vertebral variou de 0 a $15 \mathrm{~cm}(6,4 \pm 3,9)$, sendo $51,7 \%$ durante o período de tração (Tabelas 2 e 3).

\section{DISCUSSÃO}

A ocorrência de complicações como redução da função respiratória, úlceras de pressão, contraturas de partes moles e inabilidade de controle de deformidades pélvicas durante o tratamento de deformidades graves da coluna vertebral, por meio de trocas gessadas, estimulou a busca por novos métodos de tratamento ${ }^{5}$. Os primeiros relatos do halo-craniano descrevem sua utilização no tratamento da paralisia cervical associada à poliomielite por Nickel et al. ${ }^{6,7}$. A utilização do halo-craniano no tratamento das deformidades da coluna vertebral foi inicialmente descrita pelos mesmos autores ${ }^{1}$.

A utilização da tração halo-gravitacional no tratamento das deformidades da coluna vertebral baseia-se nas propriedades mecânicas dos tecidos biológicos demonstrados em estudos experimentais ${ }^{8}$. Em estudo clínico, foi demonstrado o comportamento da força de distração produzida pelas hastes de Harrington, ilustrando o relaxamento e a adaptação tempo-dependente da coluna vertebral submetida ao estresse contínuo ${ }^{8,9}$. Essa característica é denominada de viscoelasticidade.

Os casos bem sucedidos relatados pela literatura, com a utilização da tração halo-gravitacional, motivaram-nos a utilizá-la, considerando o menor risco de lesão neurológica, como visto em estudos da correção cirúrgica aguda das deformidades da coluna vertebral ${ }^{3}$. A correção cirúrgica 

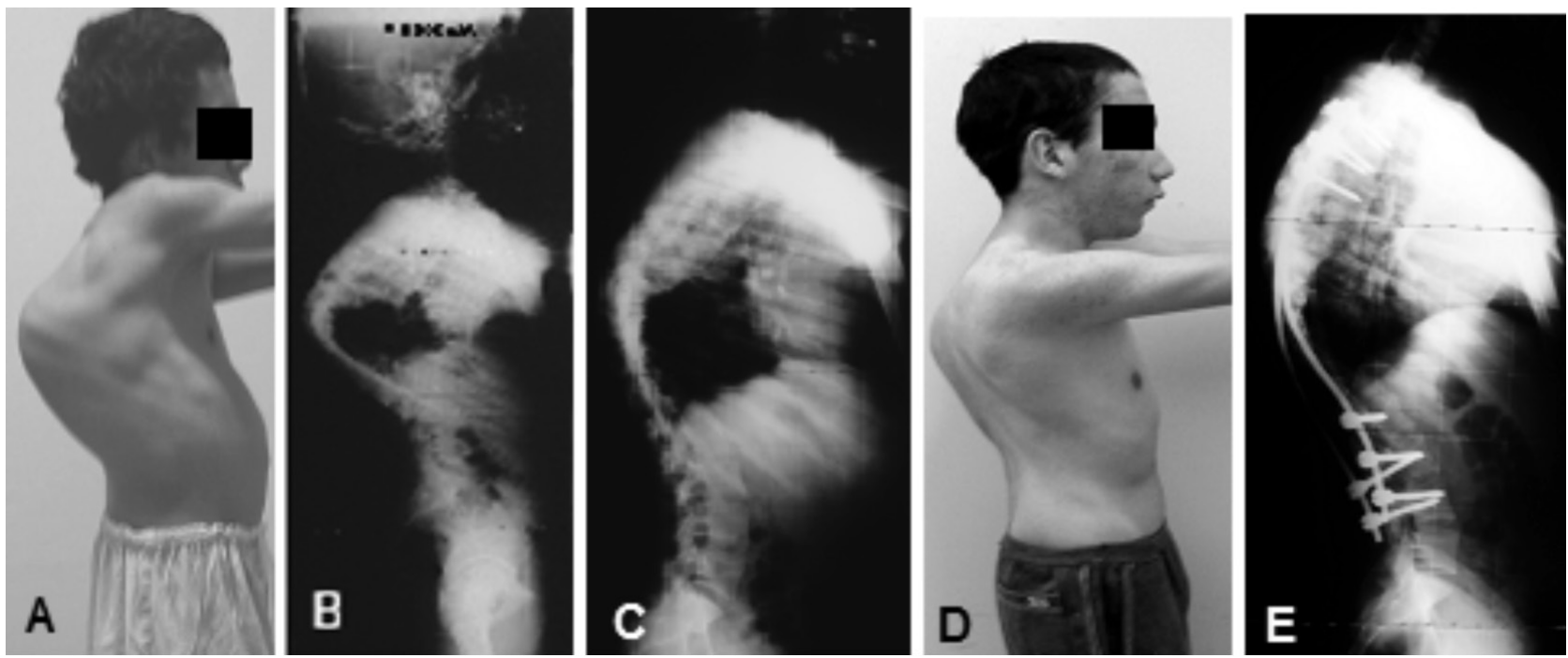

Figura 4

Fotografias pré (A e B), tração (C) e pós-operatórias (C e D) de paciente com cifose congênita (Paciente $n^{\circ 9}$ ).

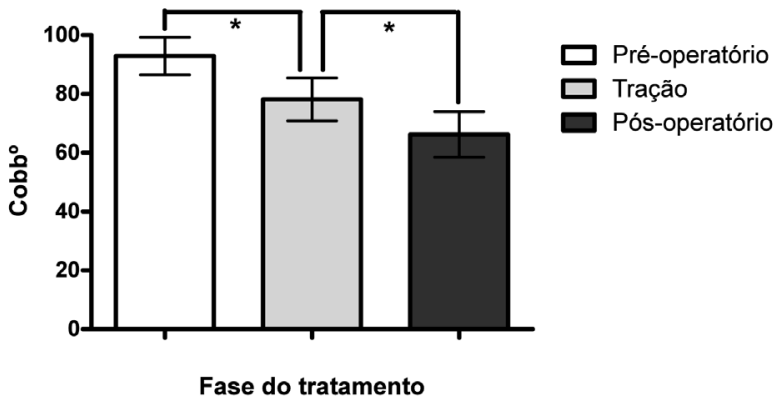

Figura 5

Representação gráfica das medidas das curvas escolióticas em diferentes fases de tratamento. $\left(^{*}\right)$ significa $p<0,0001$.

aguda das deformidades vertebrais rígidas e de alto valor angular aumenta o risco do comprometimento neurológico, em especial na presença de cifose acentuada. O intuito do halo gravitacional é promover a correção cirúrgica das deformidades vertebrais, das mais diversas patologias, de maneira mais segura, obtendo resultados similares ${ }^{1,2}$.

Com o advento de novas técnicas cirúrgicas, de sistemas de monitoração neurológica e implantes modernos, existe grande debate no que diz respeito às indicações da tração com halo-gravitacional no tratamento das deformidades graves da coluna vertebral. Osteotomias e ressecções vertebrais contribuindo para correção de grandes deformidades são realizadas com mais segurança quando associadas à análise eletrofisiológica da medula e raízes nervosas por meio de potenciais evocados. Não existe controvérsia com relação à supressão da utilização do halocraniano no tratamento de pacientes com curvas flexíveis e sem patologia intraespinhal.

A avaliação dos resultados do tratamento por meio de tração halo-craniana é complexa, pois a deformidade pode apresentar inúmeras etiologias (artrogripose, congênita, neuromuscular) tornando os parâmetros difíceis de serem comparados $^{1,2}$.

A correção obtida em nossa série de pacientes foi satisfatória, estando de acordo com outros estudos, e deve ser ressaltado o cuidado para evitar-se a comparação entre diferentes patologias ${ }^{10}$. Em um estudo foi relatada heterogeneidade nos valores angulares de correção encontrados. Nas séries em que predominam patologias congênitas ou artrogriposis foram observados valores menores quando em comparação às séries que agruparam pacientes com deformidades idiopáticas e neuromusculares ${ }^{1}$.

A controvérsia persiste acerca do protocolo ideal para a tração halo- gravitacional ${ }^{4,5,11-15}$. O número de pinos cranianos, o peso e o dia inicial, o aumento do peso e o limite máximo de peso utilizado são questões ainda debatidas na literatura. $\mathrm{O}$ protocolo utilizado em nosso estudo compreendeu a instalação do halo gravitacional com 4 pinos cranianos, início da tração com $1 \mathrm{~kg}$ no segundo dia após a instalação do halo gravitacional, o aumento do peso era de $1 \mathrm{~kg}$ por dia até a tolerância do paciente ou ausência de melhora da correção ${ }^{1,2}$.

Em nossa série, um caso de soltura do halo gravitacional foi observado após a colocação inicial de peso (paciente sete), tendo sido resolvido com nova instalação do halo. Diferente de outros estudos não foram observadas complicações como perda da lordose, degeneração cervical apofisária, alterações neurológicas, infecção intradural e extradural em nosso grupo de pacientes ${ }^{1,2,12,13,15-24}$.

\section{CONCLUSÃO}

$\mathrm{O}$ tratamento cirúrgico das deformidades graves da coluna vertebral, por meio da utilização da tração halo-gravitacional pré-operatória, mostrou-se uma boa opção de tratamento, reduzindo os valores de correção intraoperatória, diminuindo, dessa forma, os riscos de lesão neurológica durante a correção cirúrgica. 


\section{REFERÊNCIAS}

1. Rinella A, Lenke L, Whitaker C, Kim Y, Park SS, Peelle M, et al. Perioperative halo-gravity traction in the treatment of severe scoliosis and kyphosis. Spine (Phila Pa 1976). 2005;30(4):475-82.

2. Sponseller PD, Takenaga RK, Newton P, Boachie O, Flynn J, Letko L, et al. The use of traction in the treatment of severe spinal deformity. Spine (Phila Pa 1976). 2008;33(21):2305-9.

3. Stagnara P. [Cranial traction using the "Halo" of Rancho Los Amigos]. Rev Chir Orthop Reparatrice Appar Mot. 1971;57(4):287-300. [French].

4. Mubarak SJ, Camp JF, Vuletich W, Wenger DR, Garfin SR. Halo application in the infant. J Pediatr Orthop. 1989;9(5):612-4.

5. Dewald RL, Ray RD. Skeletal traction for the treatment of severe scoliosis. The University of Illinois halo-hoop apparatus. J Bone Joint Surg Am. 1970;52(2):233-8.

6. Nickel VL, Perry J, Garrett A, Heppenstall M. The halo. A spinal skeletal traction fixation device. J Bone Joint Surg Am. 1968;50(7):1400-9.

7. Nickel VL, Perry J, Garrett A, Heppenstall M. The halo. A spinal skeletal traction fixation device. By Vernon L. Nickel, Jacquelin Perry, Alice Garrett, and Malcolm Heppenstall, 1968. Clin Orthop Relat Res. 1989;(239):4-11.

8. Clark JA, Hsu LC, Yau AC. Viscoelastic behaviour of deformed spines under correction with halo pelvic distraction. Clin Orthop Relat Res. 1975;(110):90-111.
9. Nachemson A, Elfström G. Intravital wireless telemetry of axial forces in Harrington distraction rods in patients with idiopathic scoliosis. J Bone Joint Surg Am. 1971;53(3):445-65.

10.Bonnett C, Brown JC, Perry J, Nickel VL, Walinski T, Brooks L, et al. Evolution of treatment of paralytic scoliosis at Rancho Los Amigos Hospital. J Bone Joint Surg Am. 1975;57(2):206-15.

11. Rozario RA, Stein BM. Complications of halo-pelvic traction. Case report. J Neurosurg. 1976;45(6):716-8.

12.Ransford AO, Manning CW. Complications of halo-pelvic distraction for scoliosis. J Bone Joint Surg Br. 1975;57(2):131-7.

13.Ransford AO, Manning CW. Halo-pelvic apparatus: peritoneal penetration by pelvic pins. J Bone Joint Surg Br. 1978;60-B(3):404-5.

14.Tredwell SJ, O'Brien JP. Avascular necrosis of the proximal end of the dens. A complication of halo-pelvic distraction. J Bone Joint Surg Am. 1975;57(3):332-6.

15. Tredwell SJ, O’Brien JP. Apophyseal joint degeneration in the cervical spine following halo-pelvic distraction. Spine (Phila Pa 1976). 1980;5(6):497501.

16.O'Brien JP, Yau AC, Smith TK, Hodgson AR. Halo pelvic traction. A preliminary report on a method of external skeletal fixation for correcting deformities and maintaining fixation of the spine. J Bone Joint Surg Br. 1971;53(2):217-29.

17.O'Brien JP, Yau AC, Hodgson AR. Halo pelvic traction: a technique for severe spinal deformities. Clin Orthop Relat Res. 1973;(93):179-90.
18.O'Brien JP. The halo-pelvic apparatus. A clinical, bio-engineering and anatomical study. Acta Orthop Scand Suppl. 1975;163:1-188.

19.Humbyrd DE, Latimer FR, Lonstein JE, Samberg LC. Brain abscess as a complication of halo traction. Spine (Phila Pa 1976). 1981;6(4):365-8.

20.Dove J, Hsu LC, Yau AC. The cervical spine after halo-pelvic traction. An analysis of the complications of 83 patients. J Bone Joint Surg Br. 1980;62-B(2):158-61.

21.Dove J, Hsu LC, Yau AC. Spontaneous cervical spinal fusion. A complication of halo-pelvic traction. Spine. 1981;6(1):45-8.

22.Dove J, Hsu LC, Yau AC. Avascular necrosis of the dens: a followup study. Spine (Phila Pa 1976). 1982;7(5):408-11.

23. Tredwell SJ, O'Brien JP. Avascular necrosis of the proximal end of the dens. A complication of halo-pelvic distraction. J Bone Joint Surg Am. 1975;57(3):332-6.

24.Wilkins C, MacEwen GD. Cranial nerve injury from halo traction. Clin Orthop Relat Res. 1977;(126):106-10.

\section{Correspondência}

Helton LA Defino

Avenida Bandeirantes, 3900, $11^{\circ}$ andar

CEP: 14048-900

Ribeirão Preto (SP), Brasil

Fone: (16) 3602-2513

E-mail: hladefin@fmrp.usp.br 